\title{
Patient Advocacy and Changing Paradigm in Drug Access
}

\author{
Amit Dang', Vallish BN²
}

\section{Amit Dang ${ }^{1}$, Vallish BN²}

${ }^{1}$ Founder and CEO, MarksMan Healthcare Solutions, $7^{\text {st }}$ Floor, Plot No. 6, Sector $12 A$, Kopar Khairane, Navi Mumbai, Maharashtra - 400709, India. ${ }^{2}$ Assistant Professor, Department of Pharmacology, Velammal Medical College Hospital and Research Institute, Anuppanadi, Madurai - 625009. TN, India.

\section{Correspondence}

\section{Dr. Amit Dang}

Founder and CEO, MarksMan Healthcare Solutions 1st Floor, Plot No. 6, Sector 12A, Kopar Khairane, Navi Mumbai, Maharashtra - 400709, India

Ph.no: +91-7738389300

Email: amit.d@marksmanhealthcare.com

\section{History}

- Submission Date: 13-02-2016;

- Review completed: 10-09-2016;

- Accepted Date: 07-10-2016.

DOI : 10.5530/ijmedph.2016.4.2

Article Available online

http://www.ijmedph.org/v6/i4

\section{Copyright}

(C) 2016 Phcog.Net. This is an openaccess article distributed under the terms of the Creative Commons Attribution 4.0 International license.

\begin{abstract}
Background: In the process of drug development and drug approval, the pharmaceutical industry is challenged with increasing cost constraints, a growing need for real-world evidence and heavier regulatory scrutiny. Patient advocacy groups can be a valuable partner to the pharmaceutical industry in speeding up drug access. Patient advocates possess practical rather than purely scientific knowledge that is valuable for both drug development and drug approval. It was recognized quite early the patient advocacy groups and the pharmaceutical industry have similar goals. This understanding resulted in supporting patient involvement across the entire process of drug development, from discovery to USFDA approval for marketing. Support received by the patient advocacy groups include fundraising for research and development, organizing patients to expedite clinical trials, lobbying government and payer organizations to create public awareness, building support for research funding, and so on. Patient advocacy groups also back research for "orphan" diseases where there is a desperate need for new therapies. The recent success of Vertex and Sprout Pharmaceutical highlights the power of the patient voice in healthcare decision-making especially for orphan diseases. Conclusion: Working with patient groups can thus lead to a win-win situation for both the FDA and pharmaceutical industry.

Key Words: Patient Advocacy, Patient Voice, Drug Access, Health Outcomes, Drug Development
\end{abstract}

\section{INTRODUCTION}

An advocate is a person who does the act of pleading or arguing in favor of something. A patient advocate is a healthcare specialist who, as a liaison between patients and healthcare providers, argues with the best interest of the patient in view, in order to maintain or improve the quality of healthcare delivery to the patients that he/ she is representing. ${ }^{1}$ A patient advocate may be an individual or an organization. Patient advocates give a voice to patients, survivors and their carers on healthcare-related (public) forums.

Several years ago patient participation in drug approval process was uncommon. Nowadays, the involvement of patients is considered imperative in the development, review and dissemination of evidence-based knowledge on health treatments, technologies and services. In fact, substantial research and deliberation have led to the realization of value of patient and citizen involvement. This review article will provide an overview of the current state of knowledge and opinion on patient and citizen involvement in priority setting for pharmaceutical innovation.

\section{Levels of involvement}

Various approaches were proposed for patient and citizen involvement although not equally meaningful. In 1969, Arnstein published the participation ladder with the objective to categorise different sorts of participation. As shown in Figure 1, the eight rungs of the ladder included: Manipulation, Therapy, Informing, Consultation, Placation, Partnership, Delegated Power and Citizen Control. ${ }^{2}$

The Arnstein model was however criticized for its sole emphasis on power. Later INVOLVE, the national advisory group on public involvement in health research of the United Kingdom's National Institute for Health Research presented a condensed ladder of participation having the following three steps:

1. Consultation that utilized views of lay people to inform decision-making.

2. Collaboration that involved sharing of decisions about research with members of public.

3. Lay control where research was actively controlled, directed and managed by service users.

Amongst the several examples of user-controlled research reported by INVOLVE the most interesting was that carried out by Thyroid UK, small registered charity for improving diagnosis and management of patients with thyroid disorders. The research was prompted by experiences of some patients who suffered continuing problems although their blood test results were within the normal range. The experiences of these patients formed the rationale for the clinical trial that compared the accuracy of two different tests (blood and urine) in relation to people's symptoms. ${ }^{3}$ 


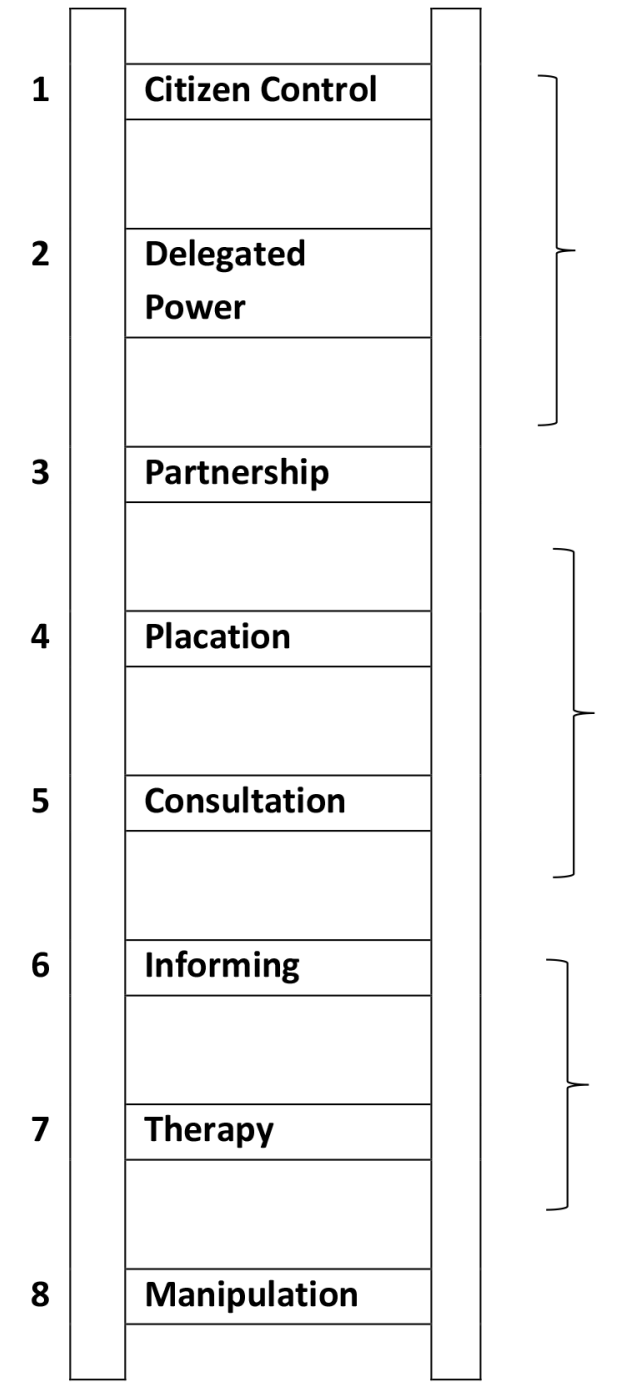

\section{Tokenism}

First step towards genuine participation.

Permits patients and citizens to have a voice.
Enables patients and citizens to negotiate and engage

in trade-offs withtraditional power holders

\section{Non participation}

People were placed on advisory committees

The main objective was to 'educate' them.

Figure 1: Arnstein's ladder of citizen participation ${ }^{2}$

\section{INSTITUTIONALIZED INVOLVEMENT: NICE, EMA AND FDA}

\section{NICE}

The National Institute for Health and Clinical Excellence (NICE) was established as a part of the British National Health Service (NHS) in 1999 with the task of guiding on which treatments or services should be provided out of public funding by the NHS and which should not. ${ }^{4}$ NICE has a fair degree of independence with respect to the following responsibility: ${ }^{3}$

- Conducting technology appraisals (including clinical and cost effectiveness);

- Guiding NHS on the use of new and existing technologies;

- Developing clinical practice and public health guidelines.

NHS staff develops NICE guidance by incorporating input from other health care stakeholders including health care professionals, patients, caregivers, industry and academics.

The three areas of health in which NICE produces guidance include: ${ }^{3}$

1. Public health,
2. Health technologies and

3. Clinical practice.

As patient and public involvement is very essential to the NICE's work it has adopted a very comprehensive approach to involving them; broadly categorized into four areas: ${ }^{3,4}$

\section{Stakeholder consultation}

Patient and caregiver organizations have the prerogative to register and comment at any stage during the clinical guideline development process. These organization can offer their inputs on guideline topics, drafting of scopes, development and initial drafting of guidelines, to the second consultation draft.

\section{Direct input}

It is mandatory to include at least two members either patients, carers or patient advocates in all NICE committees and working groups. These members are included to obtain a patient/carer perspective to the discussions and forthcoming decisions. 


\section{Indirect input}

In technology appraisal committee, the patient/carer perspective is obtained by presenting patient written testimonials or video-taped interviews.

\section{Dissemination of NICE guidance to and by patients}

It is ensured that all NICE guidance are produced in formats suitable for patients, carers and the public.

\section{NICE's Patient and Public Involvement Programme (PPIP)}

The main objective of this program is to identify patient and caregiver organizations and members of the general public willing to offer their input to NICE on guidance documents. Additionally PPIP also offers education and training programs to those patient/ consumer representatives who contribute to the guidance development process. ${ }^{4}$

\section{NICE's Citizens Council}

This council has the responsibility of integrating broad social value judgments of the general public into NICE guidance. ${ }^{4}$

\section{EMA}

The EMA is an agency of the European Union that is responsible for the scientific evaluation of medicines developed by pharmaceutical companies for use in the European Union. ${ }^{3}$ The EMA has the following scientific committees and working party wherein patient advocates participate as permanent and full members with equal voting rights: ${ }^{5}$

- Committee for Orphan Medicinal Products (COMP) since 2000.

- Patients' and Consumers' Working Party (PCWP) since 2006.

- $\quad$ Paediatric Committee (PDCO) since 2008.

- $\quad$ Committee for Advanced Therapies (CAT) since 2009.

Other activities of the patient advocates in the framework of therapeutic development include promoting drug development, ensuring clear information to patients and attempting to achieve equal access to treatments both at European and national levels. ${ }^{5}$

\section{THE U.S. FOOD AND DRUG ADMINISTRATION (FDA)}

The U.S. FDA organizes patient participation by the Patient Representative Program, Drug Development Patient Consultant Programme as well as Open Public Hearings. ${ }^{3}$

\section{Patient Representative Program}

This program is committed to offer opportunities for patients to participate in FDA decision-making. Patient representatives are recruited on an as-needed basis to advice on drugs, devices and biologics that are currently being considered for approval as well as give input earlier in the regulatory medical product development and review process. Thus this program facilitates in bringing patient voice to the discussions about new and already approved drugs and devices and policy questions. ${ }^{6}$ Further this program also invites patients and family members directly affected by a serious or life-threatening disease such as cancer or AIDS to participate in FDA Advisory Committee meetings to discuss products and treatment. This enables the FDA and the Advisory Committees to avail to the unique perspective of patients and family members directly affected by a serious or life-threatening disease. ${ }^{3}$ The patient representatives participate as both voting and non-voting members on decision by FDA concerning new drugs and medical devices approval for marketing. ${ }^{6}$

\section{Drug Development Patient Consultant Programme}

In this program the patient's perspective is incorporated into the drug development process by providing opportunity to participate in the FDA drug review regulatory process. Besides they also play active roles in meetings between the FDA and drug companies. A requirement for being a patient representatives and patient consultants is thorough knowledge about the disease and official association with any patient advocacy organization. A Patient Representative or a Patient Consultant may be self-nominated or selected by others to serve the role. ${ }^{3,6}$

\section{Open Public Hearings}

Open public hearing $(\mathrm{OPH})$ session are held in every Advisory Committee meeting wherein any interested candidate may present relevant information or views orally or in writing (21 CFR 14.25(a)). In addition to this the FDA also organizes public hearings to solicit the opinion of stakeholders. $^{3}$

FDA has recently encouraged and fostered the use of patient-reported outcome measures in clinical trials with the objective to support labelling claims in medical product development. These outcome measures include impact on quality of life or pain control. ${ }^{7}$

\section{Drivers for involving patients}

Three categories of motivation have been identified as drivers for involving patients and citizens in drug development or pharmaceutical innovation: ${ }^{3}$

1. A strong political desire to promote democratic ideals,

2. Ensure better alignment of innovation with unmet needs of patients

3. Demand for transparency and trust.

\section{Political desire to promote democratic ideals}

Way back in 2000, The Council of Europe declared in government reports, legislation as well as in statements from patient and citizen groups that an integral part of any democratic society resided in involving the public in any decision making process concerning their health care. Further results of several research highlighted the significance of values and ethical considerations in the development of health related guidelines. Thus in recommendation as these inputs from the patients and public was decided to be obtained as a priority and any decision be informed by these inputs. Apart from respecting societal values, it was observed that involving patients in pharmaceutical research enhanced the legitimacy and authenticity of studies conducted. As in any other research the inclusion of several perspectives was also regarded as essential and useful in pharmaceutical research. ${ }^{3}$ There thus began the trend towards empowering vulnerable and marginalized groups to involve actively in development of health services resulting in greater control over their own health care. Health researchers were prohibited to conduct research 'on' people as opposed to 'with' them as a mark of commitment to the principle of respect for autonomy. ${ }^{3}$ The United Kingdom (UK) encouraged public involvement in the decision making process for a majority of health care organizations and accordingly stated in their Research Governance Framework for Health and Social Care (2nd edition, 2005) that: 'Research [should be] pursued with the active involvement of service users and carers including where appropriate, those from hard to reach groups such as the homeless. ${ }^{8}$ Additionally the European Medicines Agency (EMA) identified 'a need and expectation for public bodies to listen to the views and experiences of patients who are the ones most affected by the regulatory decisions and engaging with these stakeholders gives the Agency and the public more confidence and reassurance in its outcomes.' 


\section{To meet patients' unmet needs}

The second category of motivation arose with the increasing realization that pharmaceutical innovation on several occasions did not effectively meet patient's needs. Several reasons were attributed to this lack of fulfilment of patient needs in health research system. Foremost amongst these were biases within the research professionals that favoured research on certain topics while ignoring those that were more important to recovery of patient function. The second reason was unintentional overlooking of questions vital to patient's needs. This error had its roots in the typical habit of research professionals to pay more emphasis on chronic rather than acute condition and severe but not common health problems. The third but the most important reason as noted in several studies was the difference in values system of health professional from those of patients. For example physicians who treat patients with atrial fibrillation place more value to avoidance of adverse consequences i.e. gastrointestinal bleeding of treatment. This is in sharp contrast to patients with atrial fibrillation who place more value on the avoidance of stroke as an outcome. Difference in value of health care professional and patients has been studied to exist in areas of arthritis, Alzheimer disease and mental health too. Thus the need for incorporating patient's input was strongly felt as they alone possessed the specific, relevant type of knowledge i.e. their 'experiential knowledge' that would enhance the relevance and quality of health research. The best way to shorten the distance between direct experience and interpretation to research results was engaging and involving patients in the decision making processes. Additionally this engagement would avoid emergence of distorted, inaccurate and damaging knowledge that was possible in the absence of patient involvement. ${ }^{3}$ With this background the National Institute for Health Research in the UK declared that 'involving patients and members of the public in research can lead to better research, clearer outcomes and faster uptake of new evidence. ${ }^{10}$

\section{Demand for transparency and trust.}

An analysis by the EMA concluded that the greatest benefit in promoting patient and citizen participation in scientific committee's is the increase in transparency and trust in the regulatory processes, the third category of motivation. It also leads to development of mutual respect between regulators and the community of patients. ${ }^{3}$ The above discussed motivations offered strong justification for efforts to involve patients and citizens in pharmaceutical research.

\section{Impact of patient and citizen involvement.}

The next immediate question arising out of the plethora of policies and initiatives to include patient and citizen in research priority setting is the impact of this involvement. Unfortunately very few studies have attempted to measure the impact of this involvement. Available evidence led to the categorisation of research findings into three heading viz. impact on research processes and outcomes, on patients and citizens involved and impact on healthcare professionals. ${ }^{3}$

\section{Impact on research processes and outcomes}

In a recent literature study that analysed the impact of involving patients in pharmaceutical research the following benefits were noted on research processes and outcomes. ${ }^{3,11}$

i. Increase in range of research topics;

ii. Identification of new themes;

iii. Highlighting issues of importance to patients;

iv. Pushing science forward;

v. Adoption of a more ethically acceptable research design;

vi. Improved trial consent procedures; vii. Enhanced recruitment and accrual rates;

viii. Improved quality of data on using patients as co-researchers and interviewers;

ix. Enhanced power and credibility of the findings, leading to wider and more accessible dissemination.

In an earlier study the following four themes were identified to be most impacted by public involvement in research: ${ }^{12}$

i. Increased recruitment to all types of research;

ii. In qualitative research wherein participants are asked to share their views and experiences, public involvement added value to research finding;

iii. Improved clinical trial design especially with regard to outcome measures;

iv. Benefits to all research participants.

\section{Impact on patients and citizens involved}

Studies that evaluated patient involvement in research have elicited both positive and negative responses. Positive response included personal gains in terms of increased knowledge, confidence and support from others in user groups. Some patients reported being satisfied on being heard and having their ideas being acted upon and that their experiences were of great use to others. Negative responses included feeling of being overburdened, reliving distressing memories and hearing stark medical details that left them uncomfortable. ${ }^{3}$

\section{Impact on professionals}

Advantages of patient involvement in research include a deeper understanding by researchers of patient issues. Often times this enhanced understanding prompted researchers to challenge their own beliefs and assumptions thereby contributing to better results. However some health care professionals did feel threatened and believed that patient's experiences could not outweigh academic qualifications and knowledge. This led to the development of two categories of researchers. Firstly professionals who promoted patients' expertise as valid and also considered it important in democracy. Secondly those who out of compulsion enacted out the policy of involvement. ${ }^{3}$

\section{Changing relationship between patients and pharmaceutical companies}

The relationship between patients and pharmaceutical companies has changed significantly and in multiple ways in the last few years. Five years ago pharmaceutical companies' involvement with patients group was simply restricted to supply of drugs as required by patients in the group. With the ushering in of patient-focused drug development, patient advocates are having more influence across the entire drug development continuum, from discovery and U.S. FDA approval to market. Initiatives like U.S. House of Representatives Committee on Energy and Commerce's 21st Century Cures, which is studying the gap between the science of cures and drug regulation have served as a catalyst to the gaining popularity of patient-focused drug development. ${ }^{13}$ Currently the US FDA receives advice form a dozen patient groups and trade associations on ways to improve its regulatory and administrative processes. The Prescription Drug User Fee Act, has also played an important role in expediting new drug approval. ${ }^{13}$

The November 2014 announcement by FDA asking for feedback-and especially feedback from patient groups-about its patient-centric activities, as well as patients' views about the medical product development process has led to several patient advocacy groups viz., The National Multiple Sclerosis (MS) Society, The Leukemia \& Lymphoma Society, National Kidney Foundation, Faster Cures, BIO, Parent Project Muscular 
Dystrophy, Arthritis Foundation, The National Health Council, Alpha-1 Foundation, Cancer Leadership Council, Juvenile Diabetes Research Foundation, National Psoriasis Foundation, Pharmaceutical Research and Manufacturers of America (PhRMA,), Cystic Fibrosis Foundation and Adva Med responding to FDA's solicitation. ${ }^{14}$

The advice offered by Alpha-1 Foundation is worth noting. "It is concerning that on most FDA panels that have patient representative SGEs, they are non-voting members and thus their perspective carries little weight in actual decision-making that may impact patient lives". ${ }^{14}$

This raises another important question i.e. how powerful is the patient voice and can qualitative data on patient's experiences supersede objective clinical outcomes.

\section{Patient Voice in healthcare decision-making}

The scientific community in general is highly sceptic of qualitative data and favour hard clinical outcome data over the former. Despite these scepticism the recent successes for Vertex and Sprout (see Box below) suggest how compelling patient testimonies can influence FDA recommendation. ${ }^{15,16}$

Case Study 1: Vertex Pharmaceutical likely to receive US FDA nod for Orkambi

Orkambi, is a new combination drug combining Ivacaftor with a new drug, Lumacaftor and indicated for management of patients with cystic fibrosis. In clinical studies, Orkambi, demonstrated a modest 3 percentage-point improvement in lung function after six months. No clinically meaningful improvements were observed in self-reported respiratory symptoms (cough, wheeze, congestion, sputum production, and difficulty breathing), as measured by respiratory domain of the Cystic Fibrosis Questionnaire-Revised (CFQ-R). Surprisingly 12 of the 13 FDA panel members voted in favour of Orkambi. The main reason for the positive recommendation from FDA was the wellorganized and compelling patient advocacy accompanying Vertex Pharmaceutical's presentation at the panel hearing. Seventeen patient advocates shared their experiences of taking Orkambi and claimed that the drug had a positive effect on their lives beyond what the clinical data indicated. In rare and orphan diseases where there is a desperate need for new therapies this example highlights the power of the patient voice in healthcare decision-making. ${ }^{15}$

Case Study 2: Sprout Pharmaceutical's "Little Pink Pill" another win for patient advocacy

Sprout Pharmaceutical's daily pill Flibanserin also referred to as the "Little Pink Pill" is intended to boost the female sex drive. Flibanserin was rejected twice by the FDA on the grounds of severe side-effects that far outweighed its benefits. However testimonies of women suffering from hypoactive sexual desire disorder, as well as the practitioners treating them swung the pendulum in favour of Flibanserin. The drug received recommendations by three quarters of the 24 FDA panel members. ${ }^{16}$

\section{Blurring lines between patient advocacy and Public Relation}

The Orkambi and Flibanserin case studies highlight the seemingly blurred lines between patient advocacy and public relation. It does raise doubts of slick marketing campaign gaining superiority over good science. Although the patient advocacy groups dismiss these doubts as baseless it would be helpful to introduce an element of balance and rigor into these highly charged and emotive panel hearings. ${ }^{15,16}$

\section{Collaborations for Rare Diseases}

In a panel discussion organized at the Global Genes Third Annual RARE Patient Advocacy Summit in March 2015, panellists discussed regarding collaborations among multiple stakeholders such as academic and government researchers, pharmaceutical and biotechnology companies, regulatory agencies, and patients and their families for the purpose of research and development of therapeutics for rare diseases. ${ }^{17}$

In fact a trend of rare diseases patient groups have started to emerge even in Asian countries such as China (Chinese Organization of Rare Disorders, CORD) and Japan. These patient groups have emerged after the realization that the responsibility of fighting for the rights of patients with a rare diseases most often falls on the shoulders of these patients themselves. Such patient groups are formed with an intention of interacting with the government and the pharmaceutical industry and push for orphan drug discovery policies. It has been observed that these rare disease patient groups are emerging as key stakeholders in Asia. ${ }^{18}$

\section{Patient Advocacy in India}

In India there are a number of regulatory bodies, scientific review committee, ethics committee and Non-governmental organization (NGO) that work towards patient rights and protection. Some organizations, generally non-profit focus on specific disease or aspects of healthcare. These organizations and are distinguished from patient advocates and referred to as Health advocacy organizations. Examples include Alagille Syndrome Alliance, CARES foundation, CSS patient Group and Komen Advocacy Alliance. ${ }^{16}$ These agencies sprung up with the advent of increasing number of corporate hospitals, healthcare related issues and research trials in India. Their main objective being to ensure patient's right and protection. Unfortunately none of these organizations truly qualify as a patient advocacy group. It is thus imperative that India moves towards establishing patient advocacy group that would provide special attention to patient care and protection of rights from the planning stage rather that at the troubleshooting stage. ${ }^{1}$

\section{CONCLUSION}

The role of patient advocacy in pharmaceutical innovation is gradually unveiling itself. Various models have been proposed to enhance citizen involvement in healthcare delivery system. The importance of proactive patient involvement has been realized by premier institutions such as the NICE, EMA, and FDA as well, and there have been programmes by all these institutions to enhance patient involvement in various processes such as guidance formulation and dissemination, orphan drug research and development, decision making on drug approvals, and clinical trial outcomes. Patient involvement in drug development aims to promote democratic ideals, ensure alignment of innovation with unmet needs of patients, and enhance transparency and trust in the process. Despite skepticisms from the scientific community the recent success for Orkambi and Flibanserinis highly indicative of the growing acceptance of patient-centred drug development. Thus, it is crucial to enhance the inclusion patients in all processes involved in healthcare delivery.

\section{REFERENCES}

1. Shah K, Garg S. Patient advocacy groups: need and opportunity in india Perspect CLin Res 2011;2(1):4-7. http://dx.doi.org/10.4103/2229-3485.76283; PMid:21584175 PMCid:PMC3088956.

2. Arnstein, S. A Ladder of Citizen Participation, JAIP. 1969;35(4):216-24. http:// dx.doi.org/10.1080/01944366908977225

3. Thiel G Van. Update on 2004 Background Paper, BP 8.5 Patient and Citizen Involve ment. Available at: www.who.int/medicines/areas/priority.../BP8_5Stakeholder. pdf. Accessed July 7, 2015.

4. The National Working Group on Evidence-Based Health Care (the Working Group) "Nothing About Us Without Us: Patient / Consumer Participation in Evidence-Based Health Care" Consumer Forum Summary. Available at: http:// 
www.evidencebasedhealthcare.org/images/pdf_files/EBH_Consumer_Forum_ Summary11.pdf. Accessed July 7, 2015.

5. Patient advocates involvement. Available at: http://www.eurordis.org/content/ patient-advocates-involvement. Accessed July 7, 2015

6. U.S. Department of Health and Human Services. About the Patient Representative Program. Available at: http://www.fda.gov/ForPatients/About/ucm412709.htm. Accessed July 7, 2015.

7. Klein RM. FDA's Latest Efforts in Patient-focused Drug Development ... Now in Full Swing! Available at: http://blogs.fda.gov/fdavoice/index.php/2013/06/ fdas-latest-efforts-in-patient-focused-drug-development-now-in-full-swing/. Accessed July 7, 2015

8. INVOLVE 2012. Diversity and inclusion: What's it about and why is it important for public involvement in research? Available at: www.invo.org.uk/.../2012/.../ INVOLVEDiversityandInclusionOct2012.pdf. Accessed July 7, 2015.

9. European Medicines Agency. Fifth report on the interaction with patients' and consumers' organizations. 2011.Available at: www.ema.europa.eu/docs/en GB/.../Report/2012/....WC500133475.pdf.Accessed July 7, 2015.

10. Rosenberg-Yunger ZR, Thorsteinsdóttir H, Daar AS, et al. Stakeholder involvement in expensive drug recommendation decisions: an international perspective. Health Policy. 2012;105(2-3):226-35. http://dx.doi.org/10.1016/j.healthpol.2011.12.002; PMid:22226141.

11. Barber R, Beresford P, Boote J, et al. Evaluating the impact of public involvement on research: A prospective case study. International Journal of Consumer Studies 2011;35(6):609-15. http://dx.doi.org/10.1111/j.1470-6431.2011.01017.x.
12. Staley K. Exploring Impact: Public involvement in NHS, public health and social care research. Available at: www.invo.org.uk/wp-content/uploads/.../invoNETexamples2013.pdf. Accessed July 7, 2015.

13. Cavallo J. How Pharmaceutical Companies Are Partnering With Patient Advocates to Ensure Access to Oncology Care: A Conversation With Thomas P. Sellers, MPA. Available at: http://www.ascopost.com/issues/september-1,-2014/howpharmaceutical-companies-are-partnering-with-patient-advocates-to-ensureaccess-to-oncology-care.aspx. Accessed July 7, 2015.

14. Gaffney A. How can FDA be Improved? Patient Groups Offer Ideas. Available at: http://www.raps.org/Regulatory-Focus/News/2014/12/10/20958/How-can-FDA be-Improved-Patient-Groups-Offer-Ideas/. Accessed July 7, 2015.

15. Willgoss T. The Power of the patient voice. Available at:http://www.linkedin. com/pulse/power-patient-voice-tom-willgoss-phd. Accessed July 7, 2015

16. Willgoss T. The Power of the patient voice. Part Deux: PR and Qualitative DataAvailable at: http://www.linkedin.com/pulse/power-patient-voice-part-deux-prqualitative-data-tom-willgoss-phd. Accessed July 7, 2015

17. The must-have collaborations for successful drug development. Availbale at: https://globalgenes.org/wp-content/uploads/2015/03/Drug-Development-WhitePaper-2015-FINAL.pdf. Accessed Oct 03, 2016.

18. Rare disease patient groups emerging as key stakeholders in Asia. Available at http://ipscience.thomsonreuters.com/blog/rare-disease-patient-groups-emerging-as-key stakeholders-in-asia/.

Cite this article : Dang A, Vallish BN. Patient Advocacy and Changing Paradigm in Drug Access. Int J Med. Public Health. 2016;6(4):154-9. 